\title{
Die interglazialen Kalksinterbildungen bei Hurlach nördlich Landsberg am Lech
}

\author{
Hermann Jerz \& Joachim Mangelsdorf *)
}

\author{
Pleistocene, tufa, interglacial environment, \\ sections, geophysical profiles, Th/U, absolute age, fluvial erosion \\ Bavaria, Alpenvorland.
}

\begin{abstract}
Kurzfassung: Von Hurlach nördlich Landsberg a. Lech werden Sinterkalke mit vielfältiger Ausbildung beschrieben. Sie zeichnen sich durch humose Lagen und durch eine reiche Molluskenfauna aus (vgl. Beitrag Kovanda, i. ds. Bd.). Nach der geologischen Situation und aufgrund weiterer Ergebnisse (Palynologie, Malakologie, U/Th-Datierung) besitzen sie ein interglaziales Alter (Riß/Würm).

Die Kalkabsätze sind heute isoliert, durch Seiten- und Tiefenerosion von den am östlichen Lechsteilhang austretenden Quellen abgeschnitten. Der Lech hat demzufolge hier in den letzten hunderttausend Jahren sein Steilufer bis zu 200 m weit nach E zurückverlegt.
\end{abstract}

\section{[The Interglacial Calcareous Freshwater Sediments near Hurlach North of Landsberg/Lech]}

Abstract: On the western bank of the Lech river near Hurlach there are calcareous freshwater sediments with a great variety of features. The sinterformations are associated with organic layers and they contain a rich molluscan fauna (see Kovanda, in this vol.). According to the geological situation and from further investigations (palynology, malacology, U-Th-dating) it can be concluded that they are interglacial (Riß/Würm).

The calcareous deposits are now isolated from the springs on the eastern slope of the Lech valley by lateral and deep fluvial erosion. From this we deduce that the Lech river has moved about 200 metres eastwards during the past one hundred thousand years.

\section{Einführung}

Im bayerisch-schwäbischen Grenzgebiet am Lech ist seit längerem ein bislang noch nicht näher beschriebenes größeres Vorkommen mit Sinterkalken bekannt,

*) Anschriften der Autoren: Dr. H. JerZ, Bayerisches Geologisches Landesamt, Heßstraße 128, D - 8000 München 40. Dr. J. MANGeLSDORF, Bayerisches Landesamt für Wasserwirtschaft, Lazarettstraße 67, D - 8000 München 19. das in mehrfacher Hinsicht als einzigartig bezeichnet werden kann:

- in einem Profil sind vielfältige Quellenkalke ausgebildet, mit einer arten- und individuenreichen Molluskenfauna (s. Beitrag KovANDA, in diesem Band);

- sie unterscheiden sich von den häufigen postglazialen Kalksinterbildungen im Alpenvorland durch ihr interglaziales Alter;

- sie wurden durch starke Seitenerosion des Lech im Hoch- und Spätglazial von kalkreichen Quellen abgeschnitten, aus denen sie ausgefällt wurden. Sie bildeten lange Zeit im Flußbett eine natürliche Schwelle, die kurz nach dem ersten Weltkrieg aus Gründen der Abflußbeschleunigung gesprengt wurde (It. Unterlagen des Wasserwirtschaftsamtes Weilheim i. Oby.). Bei der jüngsten Tiefenerosion wurden die Kalkabsätze durchschnitten und somit im Profil aufgeschlossen (Abb. 1).

Das Vorkommen befindet sich am linken Lechufer ca. $8 \mathrm{~km}$ nördlich Landsberg zwischen den Lechstaustufen 18 Kaufering und 19 Schwabstadl. Der direkte $\mathrm{Zu}$ gang zu dem Uferprofil verläuft auf dem „Lechweg”, der von der Bundesstraße 17 Landsberg-Augsburg in Höhe von Hurlach in Richtung Lechauen abzweigt.

Die Sinterkalke bilden am linken Flußufer eine steile Uferböschung von etwa 150-200 m Länge und 2,5-3 m Höhe. Das Liegende bildet ein schluffiger Sand über sandigem Kies, das Hangende ein grober Flußschotter. Am rechten Flußufer sind die Sinterkalke ausgeräumt.

\section{Zur geologisch-morphologischen Situation (vgl. Profil in Abb. 2):}

Der Lech besitzt nach Verlassen der Moränenlandschaft nördlich Schongau bis zu seiner Mündung in 


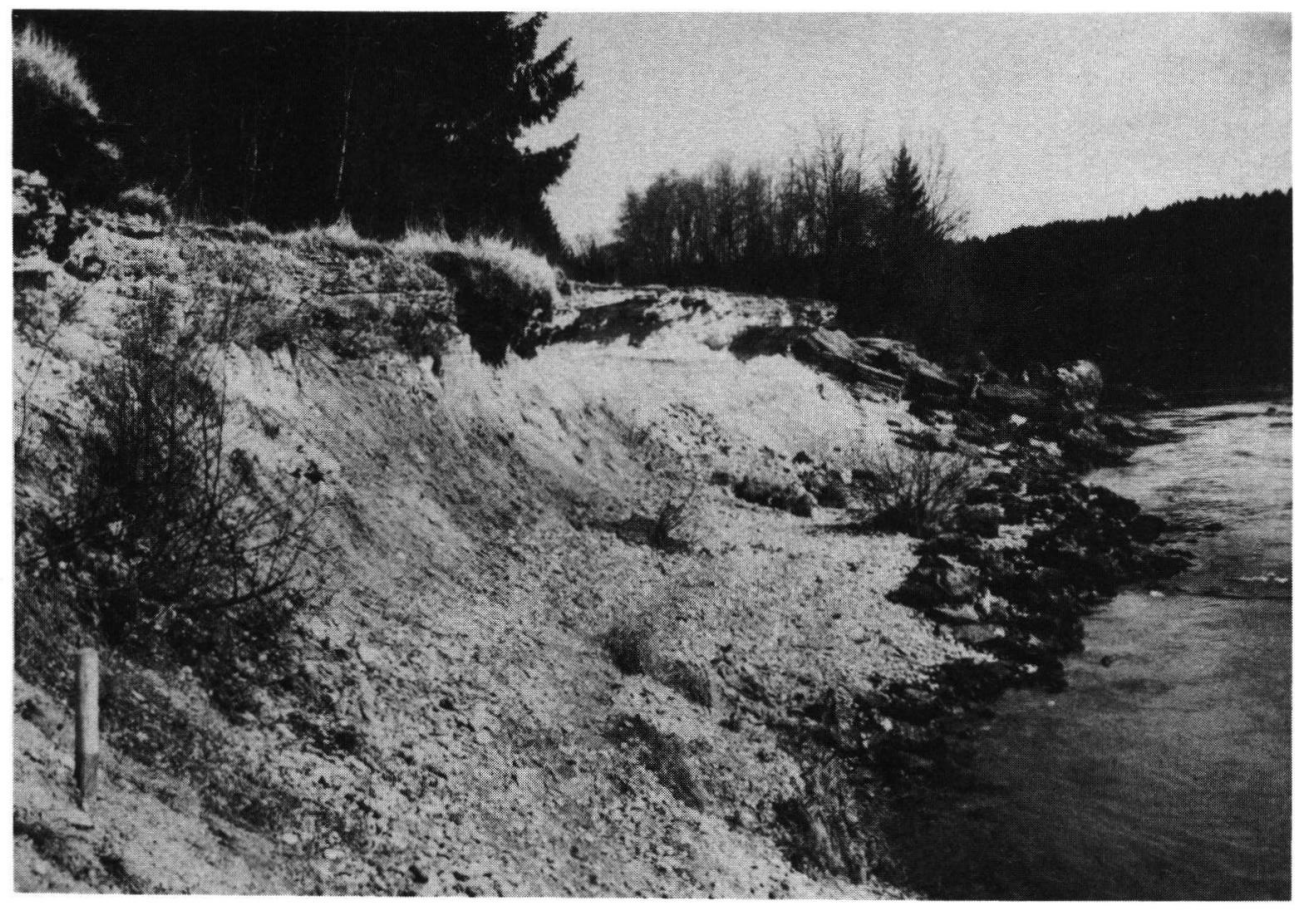

Abb. 1:

Aufschlußprofil in den Kalksinterbildungen am linken Lechufer östlich Hurlach.

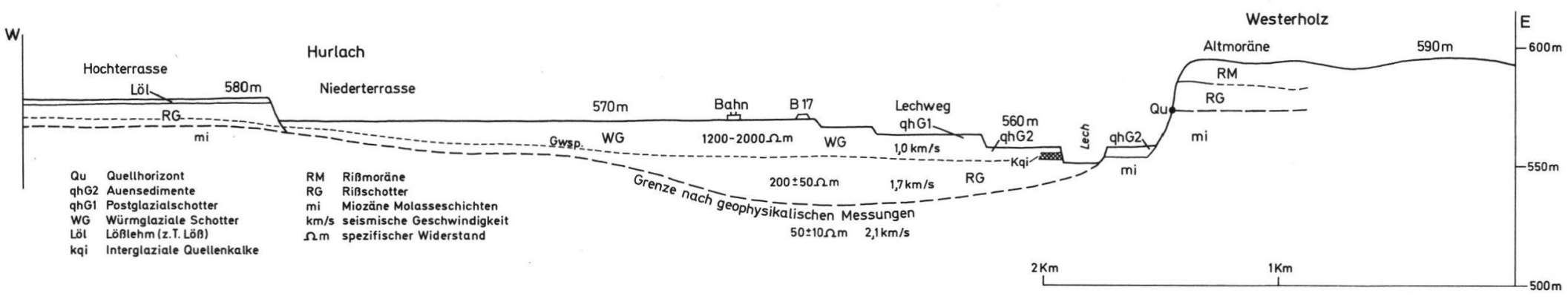

Abb. 2:

Geologisches Profil (W-E) durch das Lechtal nördlich Landsberg. 
die Donau zwei sehr unterschiedlich aufgebaute und gestaltete Flußufer. Dies gilt besonders für den $\mathrm{Ab}$ schnitt nördlich Landsberg in Höhe von Hurlach.

Den ö st li c h e n Talrand markiert ein ca. $30 \mathrm{~m}$ hohes Steilufer. Es ist in seinem oberen Teil von Moräne und Schotter (z. T. als Nagelfluh) aufgebaut, die der Rißzeit zugerechnet werden.

Im unteren Teil treten graugrüne bis graugelbe Mergel und Sande der Oberen Süßwassermolasse (Miozän) zutage. Die Grenze bei $575 \mathrm{~m}$ ü. NN wird durch einige starke Schichtquellen gekennzeichnet. Vorgelagert ist eine Auenterrasse mit jungholozänen Schottern und Sanden, die unmittelbar den Molasseschichten auflagern. Das „Tertiär” ist bei niedrigem Flußwasserstand am rechten Ufer sichtbar (vgl. Abb. 2).

Am w e s t li c h e $n$ Lechufer endet eine von Hurlach zum Lech absteigende Terrassentreppe (Hochterrasse bis Auenstufe). Sinterkalke bilden den Unterbau der jüngsten Stufe. Es ist folgendes Uferprofil aufgeschlossen (vgl. auch KovANDA 1989, in ds. Bd.):

L in kes Le chufer östlich $\mathrm{Hurlach}$ (R 4414700, H 5332650), mittlerer Abschnitt des Uferprofils

\section{$0,30(0,50) \mathrm{m}$ holozäner Flußschotter}

$0,45 \mathrm{~m}$

$0,80 \mathrm{~m}$

Moostuff mit humoser Lage, dunkelgrau

Strukturtuff aus Moos- und Algentuff, fest, glbgr, mit pflanzlichen Strukturen und Algenkrusten, teils dicht, teil dünnlagig, in langsam fließendem Wasser entstanden, sowie mit „Warzensinter” an der Unterseite von Tufflagen (epigenetische Ausfällung unter Beteiligung von Algen), stellenweise dünne humose Lagen

$0,45 \mathrm{~m} \quad$ Strukturtuff (Moos- und Algentuff) und Tuffsand mit Pflanzenabdrücken (Carex, Juncus) und Schnecken (v. a. Lymnaea)

$0,02(0,05) \mathrm{m}$ stark humoser Horizont, grauschwarz; U/Th-Datierung: rd. 120000 Jahre B.P.

$0,50 \mathrm{~m} \quad$ Kalktuffsand, mergelig, mehr oder weniger humos, mit reichlich Mollusken (große Individuen), teilweise auch Schwemmtuff, locker bis schwach verkittet, mit Algenknollen durchsetzt

$1,20 \mathrm{~m}$ Sand, schluffig, grünlichgrau, mit Mollusken

$>1,00 \mathrm{~m} \quad$ Kies, sandig.

\section{Zur zeitlichen Einstufung der Sinterbildungen}

Die Kalkausfällungen am Lech östlich Hurlach wurden zunächst als postglazial angesehen. $\mathrm{R}$ a d i o - $\mathrm{k}$ o h l e n s t off - Datierungen an zwischengeschalteten humosen Horizonten ließen erste Zweifel aufkommen. Die Altersuntersuchungen mit den nachstehenden ${ }^{14} \mathrm{C}$-Daten erbrachten ein nicht erwartetes Ergebnis (vgl. GeYH \& HeNNIG 1983: 180, Tab. 1).

Unterprofil am Lech östlich Hurlach (s. o.):

Obere humose Lage (0,9 m u. Gel.)

$16165 \pm 235$ Jahre vor 1950

Unterer humoser Horizont (rd. $2 \mathrm{~m} \mathrm{u}$. Gel.)

$30795 \pm 785$ Jahre vor 1950

$35960 \pm 1020$ Jahre vor 1950 .

Die Proben erwiesen sich als wesentlich älter als zunächst vermutet. Aus heutiger Kenntnis (s. u.) muß darüberhinaus angenommen werden, daß die Bildungen noch bedeutend älter sind und daß Infiltrationen von Huminsäuren von der Oberfläche zu kleine ${ }^{14} \mathrm{C}$ Alter verursacht haben.

Weitere Altersdatierungen mit der $\mathrm{El} \mathrm{e} \mathrm{k} \mathrm{t} \mathrm{r} \mathrm{o} \mathrm{-}$ $\mathrm{n}$ e $\mathrm{n}$ - S p in - R e s o n a n z - (E S R -) Methode an Molluskenschalen (v. a. von Lymnaea peregra), im Profil aus einer Lage zwischen 1,6 und 1,8 $\mathrm{m} \mathrm{u}$. Gel., ergaben Werte zwischen 30000 und 36000 Jahre B.P. (schriftl. Mitt. von Dr. G. J. HENNIG; vgl. auch GEYH \& HeNNIG 1983: 181). Nach dem Klimagang in den letzten 100000 Jahren und nach dem Befund, daß es sich bei den Wasserschnecken um Arten einer Warmzeit handelt, die in einem pleniglazialen Interstadial keinesfalls existieren konnten, muß auch dieses $\mathrm{Da}$ tum als zu jung angesehen werden.

$\mathrm{P}$ a $\mathrm{l}$ y no $\mathrm{log}$ is c h e Untersuchungen (Prof. Dr. E. GRÜGER, Göttingen) an einigen Proben ergaben keine für stratigraphische Vergleiche geeigneten Pollendiagramme. Trotz des hohen Humusgehaltes erwies sich die Pollenerhaltung als schlecht und die Pollendichte als gering. Die Bestimmungen ergaben aber zumindest soviel, daß die Kalkabsätze nicht in einem der pleniglazialen Interstadiale entstanden sein können. Die Pollenspektren weisen vielmehr auf Waldzeiten (mit Hainbuche) hin, wie sie in (wärmeren) Frühwürm-Interstadialen und im letzten Interglazial nachgewiesen sind. Besonders bemerkenswert ist das Fehlen von Rotbuche in allen untersuchten Proben. (Für eine Einstufung ins Postglazial müßte neben der Hainbuche auch die Rotbuche vorhanden sein.)

M a l a k o log i s c h e Untersuchungen bestätigten ebenfalls, daß es sich bei den reichlich Schneckenführenden Kalkabsätzen um Bildungen einer ausgeprägten Warmzeit handelt. Leitformen hierfür sind vor allem Aegopis verticillus (LAM.) und Pagodulina pagodula (DESM.). - (Vgl. Beitrag von J. KovandA in diesem Band). Aus dem heutigen Fehlen dieser Molluskenarten wird abgeleitet, daß die genannten 
Arten an der Fundstelle am Lech am ehesten dem letzten Interglazial angehören.

Damit im Einklang steht auch eine neuere Datierung eines Sinterkalkes mit der U / T h - M e t h o d e. Die Altersbestimmung einer Probe aus dem festen Kalktuff ca. $1 \mathrm{~m}$ u. GOK ergab ein Datum um $120300 \pm 5750$ Jahren B.P., womit letztlich ein Riß/Würm-interglaziales Alter bestätigt wird (frdl. schriftl. Mitt. von Dr. Ruth Hausmann und Dr. UDO SCHREIBER, Univ. zu Köln).

\section{Zur geologischen Situation zur Zeit der Kalksinterbildung}

Die Quellenkalke sind heute isoliert, d. h. von Quellenaustritten abgeschnitten. Nach ihrer heutigen Situation sind die Bildungsumstände nicht ohne weiteres zu erklären. Eine Lösung dieser Frage ergibt sich aus. der Rekonstruktion der damaligen geologischen Verhältnisse, insbesondere des Verlaufs der Molasseobergrenze.

G e o physika lis ch e Untersuchungen (Dr. K. BADER, Bayer. Geol. L.-Amt, München) ergaben die im Profil der Abb. 2 dargestellte Situation zwischen dem Lechufer und Hurlach: Danach fällt die Tertiärobergrenze, die am rechten Lechufer noch sichtbar ist, nach W sehr rasch ab. Die tiefste Stelle liegt im Bereich der Bundesstraße 19 bei $30-35 \mathrm{~m} \mathrm{u}$. Gel. Weiter westwärts gegen die Hochterrasse steigt die Molasse langsam bis auf $8 \mathrm{~m} \mathrm{u}$. Gel. wieder an.

Die geoelektrischen Tiefensondierungen und refraktionsseismischen Messungen lieferten sich deutlich unterscheidende spezifische Widerstände und seismische Geschwindigkeiten.

Meßprofil Hurlach - Lechweg - Lechufer:

spezifischer seismische

Widerstand Geschwindigkeit

Schotter

über dem

Grundwasser-

spiegel

$1200-2000 \Omega \mathrm{m}$

$1,0 \mathrm{~km} / \mathrm{s}$

Schotter

unter dem

Grundwasser-

spiegel

$200 \pm 50 \Omega \mathrm{m}$

$1,5-1,7 \mathrm{~km} / \mathrm{s}$

Molasse-

schichten

(Sande,

Mergel und

Tone)

$50 \pm 10 \Omega \mathrm{m} \quad 2,1-2,2 \mathrm{~km} / \mathrm{s}$
Die großen Unterschiede vor allem im spezifischen Widerstand in den Schottern sind in erster Linie grundwasserbedingt. Eine stratigraphische Grenze zwischen den oberen und den unteren Schottern läßt sich daraus nicht ablesen. Aus der geologischen Situation ergibt sich aber, daß die Sinterbildungen $(0,5$ $\mathrm{km} / \mathrm{s}$ ) rißzeitlichen Schottern auflagern und ihrerseits von postglazialen Schottern bedeckt sind.

$\mathrm{Z}$ u s a m me ngef a $\beta \mathrm{t}$ erlauben die Einzelergebnisse den Schluß, daß vor dem letzten Hochglazial das rechte Lechsteilufer sich noch im Bereich des heutigen Flußbettes befand und daß einst die Quellenkalke von den am Steilhang in halber Höhe austretenden Quellen ausgefällt wurden. Der Lech ist demnach an dieser Stelle in den letzten hunderttausend Jahren rund $200 \mathrm{~m}$ nach $\mathrm{E}$ gewandert.

Im Laufe der letzten Kaltzeit, insbesondere während des letzten Hochglazials, wurden die Sinterkalke zugeschottert. Sie kamen erst im Zuge der postglazialen Tiefenerosion wieder zum Vorschein. Erst im jüngeren Holozän wurden die Kalkabsätze vom Lech weitgehend freigespült.

\section{Schriftenverzeichnis}

BAYERISCHES LANDESAMT FÜR WASSERWIRTSCHAFT (Hrsg., 1984): 100 Jahre Wasserbau am Lech zwischen Landsberg und Augsburg. - Schriftenr. Bayer. Landesamt f. Wasserwirtschaft, 19: 126 Ș., München.

DiEZ, TH. (1968): Die würm- und postwürmglazialen Terrassen des Lech und ihre Bodenbildungen. - Eiszeitalter u. Gegenwart, 19: 102-128, 6 Abb., 6 Tab., Öhringen.

- (1973): Geologische Karte von Bayern 1: 25000, Erläuterungen zum Blatt Nr. 7931 Landsberg a. Lech. 78 S., 19 Abb., 3 Tab., München (Bayer. Geol. L.-Amt).

GeyH, M. A. \& Hennig, G. J. (1983): Datierungsversuche pleistozäner Proben aus dem Alpenvorland mit Hilfe mehrerer physikalischer Methoden. - Geologica Bavarica, 84: 177-184, 1 Abb., 3 Tab., München.

Kovanda, J. (1989): Fossile Mollusken in Kalksinterbildungen (Dauchen) am Lech-Ufer östlich von Hurlach (nördlich Landsberg/Lech). - Eiszeitalter u. Gegenwart, 39: 33-41; Hannover.

SCHreiber, U. (1985): Das Lechtal zwischen Schongau und Rain im Hoch-, Spät- und Postglazial. - Sonderveröff. Geol. Inst. zu Köln, 58: 192 S., 58 Abb., 4 Tab., 4 Beil., Köln (Selbstverl. Geol. Inst.). 